University of Nebraska - Lincoln

DigitalCommons@University of Nebraska - Lincoln

$5-15-2002$

\title{
Spin blockade effects in chromium oxide intergrain magnetoresistance
}

\author{
Andrei Sokolov \\ University of Nebraska-Lincoln, sokolov@unl.edu \\ C.S. Yang \\ University of Nebraska-Lincoln \\ Lu Yuan \\ University of Nebraska-Lincoln, lyuan2@bigred.unl.edu \\ Sy_Hwang Liou \\ University of Nebraska-Lincoln, sliou@unl.edu \\ Ruihua Cheng \\ University of Nebraska-Lincoln \\ See next page for additional authors
}

Follow this and additional works at: https://digitalcommons.unl.edu/physicsdowben

Part of the Physics Commons

Sokolov, Andrei; Yang, C.S.; Yuan, Lu; Liou, Sy_Hwang; Cheng, Ruihua; Xu, B.; Borca, C.N.; Dowben, Peter A.; and Doudin, Bernard, "Spin blockade effects in chromium oxide intergrain magnetoresistance" (2002).

Peter Dowben Publications. 20.

https://digitalcommons.unl.edu/physicsdowben/20

This Article is brought to you for free and open access by the Research Papers in Physics and Astronomy at DigitalCommons@University of Nebraska - Lincoln. It has been accepted for inclusion in Peter Dowben Publications by an authorized administrator of DigitalCommons@University of Nebraska - Lincoln. 


\section{Authors}

Andrei Sokolov, C.S. Yang, Lu Yuan, Sy_Hwang Liou, Ruihua Cheng, B. Xu, C.N. Borca, Peter A. Dowben, and Bernard Doudin 


\title{
Spin blockade effects in chromium oxide intergrain magnetoresistance
}

\author{
A. Sokolov, C.-S. Yang, L. Yuan, S.-H. Liou, Ruihua Cheng, B. Xu, C. N. Borca, \\ P. A. Dowben, and B. Doudin a) \\ Center for Materials Research and Analysis and the Department of Physics and Astronomy, Behlen \\ Laboratory of Physics, University of Nebraska, Lincoln, Nebraska 68588-0111
}

\begin{abstract}
$\mathrm{CrO}_{2}$ thin films with crystallites of a significant size permit investigation of the thin intergrain conduction between a limited numbers of crystals. Phoemission techniques reveal that the $\mathrm{CrO}_{2}$ films are covered by a 1-2-nm-thick $\mathrm{Cr}_{2} \mathrm{O}_{3}$ insulating barrier. The electronic band gap of the surface electrons is $3.4 \mathrm{eV}$ at low temperatures, decreases sharply down to $2.8 \mathrm{eV}$ at ambient. Electric transport though a few junctions in series shows a remarkable zero-bias anomaly, interpreted in terms of blockade effects. We show that the magnetoresistance is governed by low-bias blockade in these junctions. (C) 2002 American Institute of Physics. [DOI: 10.1063/1.1452240]
\end{abstract}

\section{INTRODUCTION}

Chromium oxide is among the best candidates for a high-spin polarization material. ${ }^{1}$ Such a property, with one spin direction of the conduction electrons strongly dominant, is desirable for applications in spin electronics. $\mathrm{CrO}_{2}$ pressed powders and $\mathrm{CrO}_{2} / \mathrm{Cr}_{2} \mathrm{O}_{3}$ composites showed remarkable magnetoresistance (MR) of up to $50 \%$ at low temperature. ${ }^{2-4}$ The results were explained in terms of intergrain tunneling, and the large MR values were attributed to the high degree of spin polarization of $\mathrm{CrO}_{2}$. Evidence of the large spin polarization of $\mathrm{CrO}_{2}$ has been suggested by the superconducting point contact spectroscopy method, ${ }^{5}$ spin-polarized photoemission, ${ }^{6}$ and vacuum tunneling, ${ }^{7}$ but the interpretation of the results needs to take into account the limited wave vector sampling, strong surface effects, and differences in the definitions of spin polarization. ${ }^{8}$ Discrepancies with the expected high spin polarization value are apparent in the small (1\%) MR, found on $\mathrm{CrO}_{2}$ tunnel junctions at $70 \mathrm{~K},{ }^{9}$ or the small negative values $(-8 \%)$ found at $4 \mathrm{~K}^{.10}$

We present a combination of photoemission and transport measurements demonstrating spin blockade effects in chromium oxide samples. Complementary photoemission and inverse photoemission studies confirm the presence of $\mathrm{Cr}_{2} \mathrm{O}_{3}$ insulating oxide at the surface, consistent with the studies of Dai et al. ${ }^{11}$ on commercial $\mathrm{CrO}_{2}$ powders. We show that the apparent density of states at the conduction band edge is strongly temperature dependent. The remarkable decrease of the conduction band edge energy at high temperatures can be related to low temperature anomalies in the $I-V$ curves of our junctions. A model of Coulomb blockade at the surface of the $\mathrm{CrO}_{2}$ crystal allows us to explain both types of measurements. We show that the lowtemperature magnetoresistance found in these samples decreases by a factor of 2 when the voltage bias is increased.

\section{SAMPLE PREPARATION}

Polycrystalline $\mathrm{CrO}_{2}$ films were made by rf sputtering of $\mathrm{CrO}_{3}$ onto $\mathrm{LaAlO}_{3}$ substrates and annealing in a high-

\footnotetext{
${ }^{a)}$ Author to whom correspondence should be addressed.
}

pressure cell. The sputtering was performed under of 10 mTorr argon and 2 mTorr oxygen pressure. The target was prepared by pressing $\mathrm{CrO}_{3}$ powder and sintering at $150{ }^{\circ} \mathrm{C}$. Thickness of $\mathrm{CrO}_{2} / \mathrm{CrO}_{3}$ films ranged between 0.1 and 1 $\mu \mathrm{m}$. Annealing in about $100 \mathrm{~atm}$ of oxygen pressure at $390{ }^{\circ} \mathrm{C}$ leads to the formation of the stable $\mathrm{CrO}_{2}$ phase. ${ }^{2,12}$ $\mathrm{X}$-ray diffraction confirmed the presence of $\mathrm{CrO}_{2}$, with no detectable diffraction peaks from $\mathrm{Cr}_{2} \mathrm{O}_{3}$. The thinnest annealed films showed a grain density of about 10 crystals every $100 \mu \mathrm{m}^{2}$ area. The grains were of elongated shapes, typically $5 \mu \mathrm{m}$ long and $0.5 \mu \mathrm{m}$ wide.

\section{RESULTS AND DISCUSSION}

Appropriate surface preparation was established by x-ray photoemission (XPS), ultraviolet photoemission, and inverse photoemission. The surface composition was determined by angle-resolved XPS, using the $\operatorname{Mg} K_{\alpha}$ line radiation (1253.6 $\mathrm{eV}$ ). A negligible amount of $\mathrm{C}$ (less than $3 \%$ of a monolayer), was taken as a good indicator of a clean surface following sputtering and annealing. The binding energy of $\mathrm{O} 1 \mathrm{~s}$ core level of $531.1 \pm 0.2 \mathrm{eV}$ and a significant shoulder around $529.5 \pm 0.2 \mathrm{eV}$ were found. This indicates a thin $\mathrm{Cr}_{2} \mathrm{O}_{3}$ layer formed on the surface of the samples, confirming other similar measurements. ${ }^{11}$ Angle-resolved XPS was used to characterize the thickness of the $\mathrm{Cr}_{2} \mathrm{O}_{3}$ surface layer as has been undertaken for other oxide surfaces. ${ }^{13}$ The ratio of $\mathrm{Cr}_{2} \mathrm{O}_{3}$ intensity to $\mathrm{CrO}_{2}$ intensity for each emission angle was derived by decomposing every $\mathrm{O} 1 s$ spectrum into two peaks, corresponding to the two oxide phases. We find that the thickness of the $\mathrm{Cr}_{2} \mathrm{O}_{3}$ layer is about twice the oxygen core level photoelectron mean free path, using a summation modeling analysis described elsewhere. ${ }^{13}$ This corresponds to approximately $2 \mathrm{~nm}$ thickness. ${ }^{14}$

The energy distribution curves of the valence bands were acquired at normal emission, using a $\mathrm{He} \mathrm{I}$ incident radiation source $(21.2 \mathrm{eV})$. The inverse photoemission spectra were obtained by using variable energy electron and UV detector (a Geiger-Müller detector). The overall energy bandwidth was $\sim 450 \mathrm{meV}$. The conduction band spectra were taken by changing the kinetic energy of the incidence electron energy from 5 to $19 \mathrm{eV}$. The Fermi level was established from tan- 


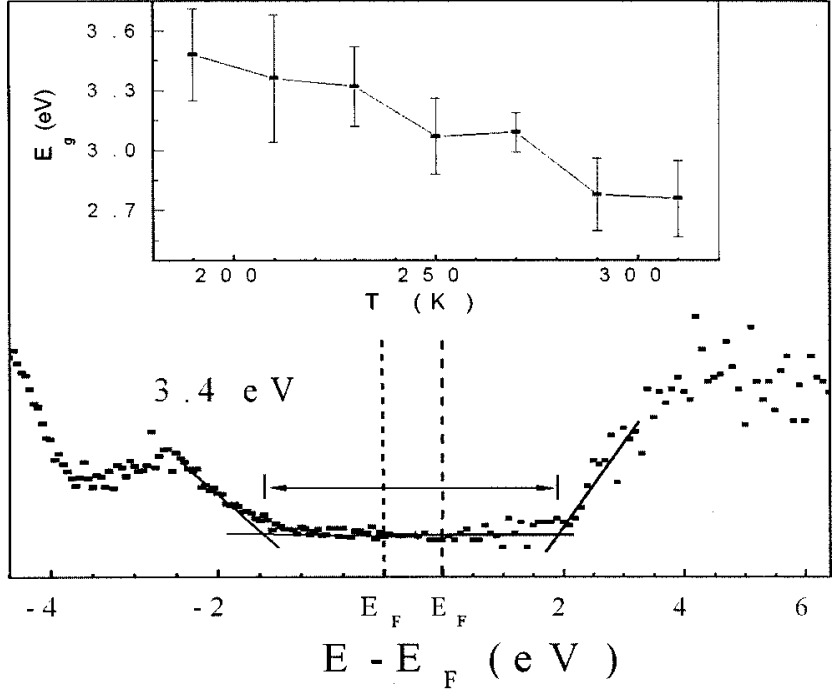

FIG. 1. The combined photoemission (left) and inverse photoemission (right) at several selected temperatures for $\mathrm{Cr}_{2} \mathrm{O}_{3}$ surface layers on $\mathrm{CrO}_{2}$. The effective gap, uncorrected for instrumental resolution, is indicated. While the photoemission and inverse photoemission features are largely temperature independent, the density of states at the conduction band edge is strongly temperature dependent, resulting in an increasingly sharp conduction band edge at high energies with decreasing temperatures. The photoemission and inverse photoemission were obtained for normal emission and normal incidence, respectively.

talum in electrical contact with the sample for both photoemission and inverse photoemission, as has been undertaken on surface studies for other potential half-metallic systems. ${ }^{15,16}$ Spectra of the density of states, near the Fermi level, of the $\mathrm{Cr}_{2} \mathrm{O}_{3}$ surface of the $\mathrm{CrO}_{2}$ sample show temperature dependence much greater than $3 k_{B} T$ (Fig. 1). At temperatures lower than $200 \mathrm{~K}$, a gap of $3.5 \pm 0.2 \mathrm{eV}$ was found (or $3.8 \pm 0.2 \mathrm{eV}$ correcting for the combined instrumental line width), confirming that the $\mathrm{Cr}_{2} \mathrm{O}_{3}$ coverage of a $\mathrm{CrO}_{2}$ grain provides a wide band gap tunnel barrier. Heating the sample to room temperature showed a remarkable decrease of the conduction band edge binding energy, without other significant changes in the spectra. An unambiguous temperature dependent change of the valence band conduction band gap by $0.7 \mathrm{eV}$ with a nonsystematic error estimate of $\pm 0.2 \mathrm{eV}$ was found between 190 and $300 \mathrm{~K}$. This change, much larger than $k_{B} T$, might be explained in terms of surface charging effects of the native oxide surface ${ }^{15}$ However, we did not find any noticeable band shifts in our spectra and the absence of any other significant changes in the spectrum, as well as the absence of a temperature discontinuity, make the hypothesis of surface phase transition very unlikely.

Electric transport measurements using two or four points ac or dc were undertaken, with electrical connections separated by less than $200 \mu \mathrm{m}$. With samples of resistance larger than a few hundreds of ohms, a strong nonlinear $I-V$ behavior was found, diminishing with increasing temperature (Fig. 2). This low-bias conductivity is strongly reminiscent of the so-called giant resistance peak observed by Rowell and Shen on $\mathrm{Cr}-\mathrm{I}-\mathrm{Ag}$ tunnel junctions. ${ }^{17}$ This insulator "I" was fabricated by oxidation of a $\mathrm{Cr}$ film, and the anomaly attributed

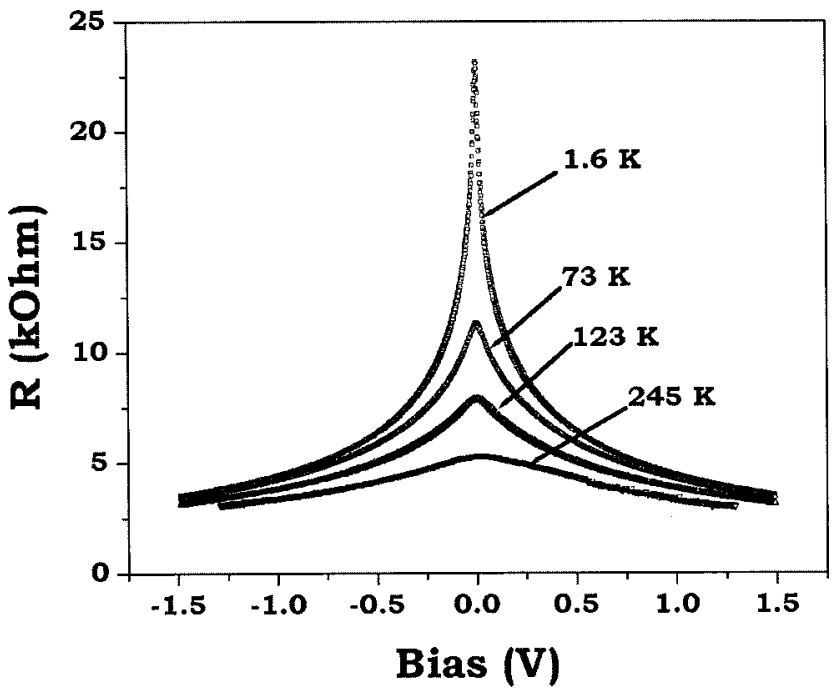

FIG. 2. Resistance vs bias at several temperatures. The sample corresponds to approximately 20 junctions in series.

to the presence of magnetic $\mathrm{CrO}_{2}$ and $\mathrm{Cr}_{2} \mathrm{O}_{3}$ ! We can directly superpose our resistance versus bias curve to the Rowell and Shen results if we scale down our bias voltage scale by a factor of 10 . The half-width of the giant resistance peak at $3 \mathrm{~K}$ is around $50 \mathrm{mV}$, which is 10 times larger than Rowell and Shen results (Fig. 1 in Ref. 17). Explanations for the giant resistance peak were given by Giaver and Zeller in their seminal paper on Coulomb blockade in tunnel junctions, ${ }^{18}$ where the low-bias peak corresponds to the current blocking due to the electrostatic charging energy of an island in the tunnel junction.

A magnetoresistance ratio, defined as $\left(R_{\max }-R_{\min }\right)$ $/ R_{\max }$, reached values of $30 \%$ at $1.6 \mathrm{~K}$, consistent with previous studies (Fig. 3). The maximum of the resistance occurred at applied field values of $150 \mathrm{Oe}$, which is the coercive force of the $\mathrm{CrO}_{2}$ films. For comparison, magnetoresistance curves for samples of low resistance values, obtained from thick films (several microns) of chromium oxide, showed similar ratios, but with more difficulty to reach saturation. The magnetoresistance decreases strongly with applied bias, on a voltage scale corresponding

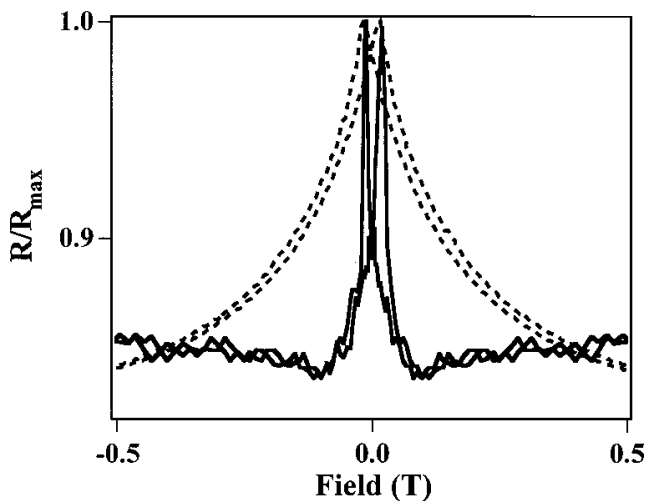

FIG. 3. Magnetoresistance curves of a chromium oxide powder. The full line curve corresponds to a sample made of a few crystallites with only a resistance of more than $10^{5} \Omega$. The shaded line curve corresponds to a thick film sample (several microns), with a resistance of less than $10 \Omega$. 


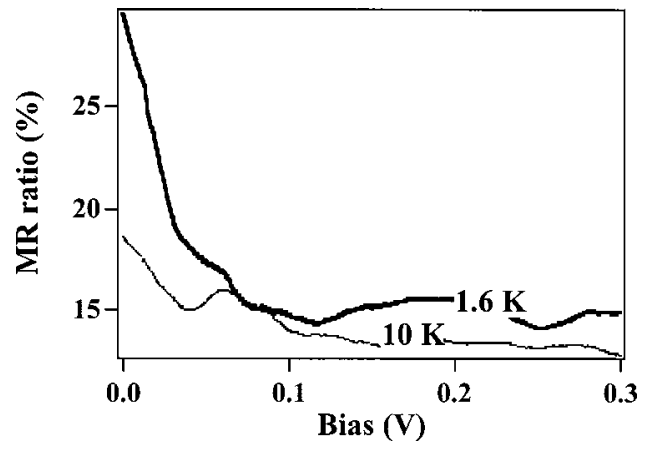

FIG. 4. Magnetoresistance of $\mathrm{CrO}_{2} / \mathrm{Cr}_{2} \mathrm{O}_{3} / \mathrm{CrO}_{2}$ junctions vs applied bias at low temperatures. The zero bias enhancement appears clearly at temperatures lower than $10 \mathrm{~K}$.

to the occurrence of the zero-bias anomaly (Fig. 4). Such behavior is not observed for low resistance samples, where a large number of junctions are measured in series.

The interplay between Coulomb blockade and tunnel magnetoresistance (TMR) has recently attracted a lot of interest, mostly motivated by the perspective of the enhancement TMR by spin blockade effects. Theoretical models involving cotunneling ${ }^{19}$ and spin accumulation ${ }^{20,21}$ have been put forward. The magnetoresistance behavior in Fig. 4 corresponds to the predictions of Takahashi and Maekawa, using a model of enhancement of MR due to cotunneling effects. ${ }^{19}$ At low-bias voltage values, the current flows though virtual states in the barrier. ${ }^{22}$ The correlated hopping events lead to a squared magnetoresistance value, or a doubling of the magnetoresistance ratio. At higher bias, the magnetoresistance value saturates at half its zero-bias value. Data in Fig. 4 reproduces such a prediction remarkably well.

\section{CONCLUSIONS}

Studies on samples made of a few crystals allowed us to characterize the nonlinear $\mathrm{CrO}_{2}$ intergrain conductivity, which includes $\mathrm{Cr}_{2} \mathrm{O}_{3}$ barrier layers, without resorting to the investigation of the $\mathrm{CrO}_{2} / \mathrm{Cr}_{2} \mathrm{O}_{3}$ composite system. Current-voltage measurements reveal the zero bias anomaly of these junctions, which is not observed when many junctions are measured in series. Angle-resolved XPS investigations performed on the same samples showed a $\mathrm{Cr}_{2} \mathrm{O}_{3}$ cov- erage to be more than $1 \mathrm{~nm}$ in thickness. This insulating surface oxide also plays the role of a thin barrier between adjacent crystallites. Combining photoemission data and current-voltage measurements, a picture of Coulomb blockade in these junctions emerges. It explains the high magnetoresistance values at low bias. Chromium oxide systems therefore show an enhancement of the magnetoresistance due to imperfections in the insulating barrier, playing the role of a blockade for electron flow.

\section{ACKNOWLEDGMENTS}

The support of the NSF CAREER Program (Grant No. DMR 98-74657), the NSF (DMR 98-02126), the Office of Naval Research, and the Nebraska Research Initiative are gratefully acknowledged.

${ }^{1}$ H. van Lueken and R. A. de Groot, Phys. Rev. B 51, 7176 (1995).

${ }^{2}$ H. Y. Hwang and S.-W. Cheong, Science 278, 1607 (1998).

${ }^{3}$ J. M. D. Coey, A. E. Berkowitz, L. Balcells, and F. F. Putris, Phys. Rev. Lett. 80, 3815 (1998).

${ }^{4}$ S. S. Manoharan, D. Elefant, G. Reiss, and J. B. Goodenough, Appl. Phys. Lett. 72, 984 (1998).

${ }^{5}$ R. J. Soulen et al., Science 282, 85 (1998).

${ }^{6}$ K. P. Kämper, W. Schmitt, G. Güntherodt, R. J. Gambino, and R. Ruf, Phys. Rev. Lett. 59, 2788 (1987).

${ }^{7}$ R. Weisendanger, H.-J. Güntherodt, G. Güntherodt, R. J. Gambino, and R. Ruf, Phys. Rev. Lett. 65, 247 (1990).

${ }^{8}$ I. I. Mazin, Phys. Rev. Lett. 83, 1427 (1999)

${ }^{9}$ A. Barry, J. M. D. Coey, and M. Viret, J. Phys.: Condens. Matter 12, L173 (2000).

${ }^{10}$ A. Gupta, X. W. Li, and Gang Xiao, Appl. Phys. Lett. 78, 1894 (2001).

${ }^{11}$ J. Dai, J. Tang, H. Xu, L. Spinu, W. Wang, K.-Y. Wang, A. Kumbhar, M. Li, and U. Diebold, Appl. Phys. Lett. 77, 2840 (2000).

${ }^{12}$ B. L. Chamberland, CRC Crit. Rev. Solid State Mater. Sci. 7, 1 (1977).

${ }^{13}$ Jaewu Choi, J. Zhang, S.-H. Liou, P. A. Dowben, and E. W. Plummer, Phys. Rev. B 59, 13453 (1999).

${ }^{14}$ R. Cheng, B. Xu, C. N. Borca, A. Sokolov, C.-S. Yang, L. Yuan, S.-H. Liou, B. Doudin, and P. A. Dowben, Appl. Phys. Lett. 79, 3122 (2001).

${ }^{15}$ H. Dulli, E. W. Plummer, P. A. Dowben, J. Choi, and S.-H. Liou, Appl. Phys. Lett. 77, 570 (2000).

${ }^{16}$ D. Ristoiu, J. P. Nozières, C. N. Borca, T. Komesu, H.-K. Jeong, and P. A. Dowben, Europhys. Lett. 49, 624 (2000).

${ }^{17}$ J. M. Rowell and L. Y. L. Shen, Phys. Rev. Lett. 17, 15 (1966).

${ }^{18}$ I. Giaver and H. R. Zeller, Phys. Rev. Lett. 20, 1504 (1968).

${ }^{19}$ S. Takahashi and S. Maekawa, Phys. Rev. Lett. 80, 1758 (1998).

${ }^{20}$ J. Barnas and A. Fert, Phys. Rev. Lett. 80, 1058 (1998).

${ }^{21}$ A. Brataas, Y. V. Nazarov, J. Inoue, and G. E. Bauer, Phys. Rev. B 59, 93 (1999).

${ }^{22}$ D. V. Averin and Yu V. Nazarov, Phys. Rev. Lett. 65, 2446 (1990). 